\title{
Efficient Shape Priors for Spline-Based Snakes
}

\author{
Ricard Delgado-Gonzalo, Daniel Schmitter, Virginie Uhlmann, and Michael Unser, Fellow, IEEE
}

\begin{abstract}
Parametric active contours are an attractive approach for image segmentation, thanks to their computational efficiency. They are driven by application-dependent energies that reflect the prior knowledge on the object to be segmented. We propose an energy involving shape priors acting in a regularization-like manner. Thereby, the shape of the snake is orthogonally projected onto the space that spans the affine transformations of a given shape prior. The formulation of the curves is continuous, which provides computational benefits when compared with landmark-based (discrete) methods. We show that this approach improves the robustness and quality of splinebased segmentation algorithms, while its computational overhead is negligible. An interactive and ready-to-use implementation of the proposed algorithm is available and was successfully tested on real data in order to segment Drosophila flies and yeast cells in microscopic images.
\end{abstract}

Index Terms-Active contours, model-based segmentation, parametric snake, deformable template, B-spline, shape space.

\section{INTRODUCTION}

$\mathbf{S}$ HAPE recognition is an active field of research with a widespread area of application that includes domains such as biology, medicine, or computer vision. In order to recognize a shape, a suitable shape description and a measure of fit have to be defined. This allows one to compare the segmented structure to a reference. The description can be either discrete, typically involving landmarks [1], or can entail some form of continuous-domain processing [2]. In general, the characterization and detection of shapes is facilitated when prior knowledge is available.

In this paper, we present a method for the shape recognition of closed curves that minimizes a measure of distance with respect to a reference shape, up to an affine transformation. We define as "affine shape space" the space that contains all planar closed curves that are affine transformations of the reference shape. Then, an essential step of our method is the analytical derivation of the orthogonal projection of any curve onto the shape space thus defined.

We describe the process of shape recognition as the evolution of a parametric active contour (a.k.a. snake) [3]-[5]. Active contours are widely used

Manuscript received July 14, 2014; revised January 30, 2015, May 19, 2015, and June 28, 2015; accepted July 10, 2015. Date of publication July 16, 2015; date of current version July 30, 2015. This work was supported in part by the Swiss SystemsX.ch Initiative under Grant 2008/005 and in part by the Swiss National Science Foundation for the Promotion of Scientific Research under Grant 200020-121763 and Grant 200020-144355. The associate editor coordinating the review of this manuscript and approving it for publication was Dr. Nilanjan Ray.

The authors are with the Biomedical Imaging Group, École Polytechnique Fédérale de Lausanne, Lausanne 1015, Switzerland (e-mail: ricard.delgado@epfl.ch; daniel.schmitter@epfl.ch; virginie.uhlmann@epfl.ch; michael.unser@epfl.ch).

Color versions of one or more of the figures in this paper are available online at http://ieeexplore.iee.org.

Digital Object Identifier 10.1109/TIP.2015.2457335 to segment images. They are curves that evolve from an initial position towards a boundary of interest in order to enclose the object to be segmented. Different variants of snakes have been proposed in the literature [6]-[10]. There, the evolution of the contour is typically driven by the minimization of a certain energy term [11].

We parameterize continuously the closed curve by means of (exponential) B-spline basis functions [11]-[13] and their corresponding control points; this representation was proved to be effective for fast energy minimization. Additionally, B-splines provide a convenient way to handle intrinsic shape properties of the curve, such as smoothness constraints [14]. The spline-based parameterization allows us to fully define a closed curve by its control points only, while preserving all the information of the continuous trajectory of the curve. Therefore, the projection of an arbitrary spline curve onto the shape space can be expressed as a function of the control points that is based on a continuously defined distance between a curve and the shape space.

Our formulation encapsulates the complexity derived from the use of continuously defined curves. Other methods based on discrete or semi-discrete approaches exist. For instance, landmark-based methods minimize the distance of a curve (discrete or continuous) to a set of points on the plane [15]. These methods are not computationally intensive since the number of operations is essentially proportional to the number of landmarks. However, the energy they minimize may be biased because the landmarks are usually not uniformly distributed in space. Thus, regions with accumulation of landmarks get more attention from the optimization algorithms. Other popular methods are the pixel- or grid-based methods, where curves are intrinsically discretized within the pixel grid [16]. The computational cost of such methods is dominated by the number of pixels used to discretize the curves. This approach becomes computationally expensive in highresolution images, where a large amount of pixels is needed. In this case, the energy is biased again due to an uneven distribution of distances within a pixel-grid, which introduce quantification artefacts working against the optimizer. Solutions for including prior shape knowledge in geodesic active contour models can be found in the literature [17]-[19]. Foulonneau et al. [20] propose a snake energy using shape priors based on affine transformations. However, it can only be used for region-based energies and also requires a prior normalization step, which can introduce a bias into the framework. Charmi et al. [21] propose shape priors based on the similarity transform; a different class of transforms than the affine transformations. Furthermore, their method is based on either discrete curves or level sets while also including a prior normalization step. 
Our alternative proposal is to define the orthogonal projector onto the shape space that is given by a reference spline curve and all its affine transformations. This implicit definition of a shape space allows us to define a snake energy term including prior knowledge. No normalization step is used and the energy term can be formulated in the continuous domain. However, by using a spline-based description of the parametric curves, the proposed solution can be implemented with no additional cost compared to curves defined by a discrete set of landmarks. To our knowledge this approach has not been proposed in an active contour framework so far.

The main contributions of this paper are:

1) Presentation of a shape projector that maps an arbitrary continuously-defined spline curve onto the shape space.

2) Proposition of an analytic expression of a distance measure for the shape recognition of closed spline curves using shape priors.

3) A spline-based continuous-domain solution that can be implemented with no additional computational cost compared to the approach where curves are described by a discrete set of points, which allows to be computationally efficient.

4) A ready-to-use implementation of the proposed framework that is freely available in the public domain as a plug-in for the bioimage-analysis platform Icy [22].

The main field of application of our proposed method is the segmentation of biological objects such as cells, bacteria or other model organisms within images. Thereby, a sketch of the outline of the structure of interest to be segmented in a particular image is known beforehand. Often size, orientation location and other aspects that can be described by affine transformations are to be estimated in such settings. If the class of possible transformations can be restricted to be affine, prior knowledge can be used to enhance robustness of the segmentation result. The choice of using spline-curves has the advantage that user-interaction can be implemented by allowing the user to locally change the curve by moving its control points; a property that is often appreciated by final users within the life-sciences community.

This paper is organized as follows: In Section II, we present a review of the parametric snake model and the associated framework. In Section III, we introduce the shape space and derive the corresponding projector. Then, we describe in Section IV how to incorporate prior shape information to parametric snakes algorithms. In Section V we perform several experiments in order to study the validity of our prior shape energy term. We also show its usefulness in real experimental conditions on two different biological image sets. Finally, in Section VI, we describe the implementation details and availability of the software implementation of our work.

\section{Spline-Based SNAKeS}

\section{A. Parametric Representation of Closed Curves}

Let $\mathbf{r}: D \rightarrow \mathbb{R}^{2}$ be a parametric curve on the plane, i.e., $\mathbf{r}(t)=\left(r_{1}(t), r_{2}(t)\right)$ with $t \in D$ a continuous parameter. Since $\mathbf{r}$ is closed, the two 1D coordinate functions $r_{1}$ and $r_{2}$ are periodic and share the same period. For simplicity, (a)

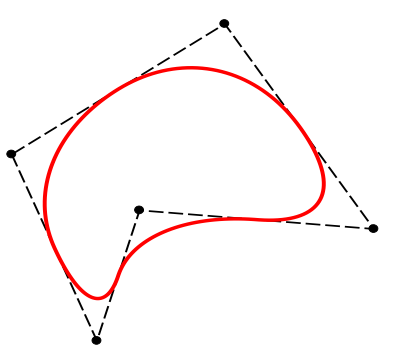

(b)
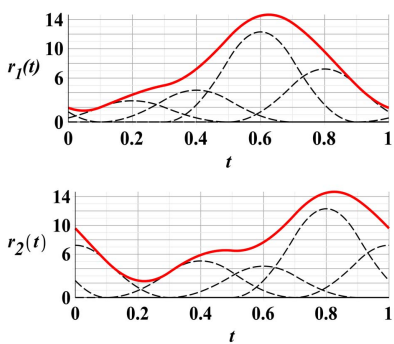

Fig. 1. Parametric representation of a closed curve in spline-based snake models. (a) The parametric model for describing closed curves is given in (1) $(M=5)$. The contour of the spline-based snake is shown as a solid line. The control points $\{\mathbf{c}[k]\}_{k \in \mathbb{Z}}$ of the snake model are represented by dots joined by a dashed polygon (a.k.a. the control polygon). (b) The parametric functions $r_{1}(t)$ and $r_{2}(t)$ are displayed as solid lines, and the dashed lines indicate the weighted basis functions.

we normalized this period to be unity. Under these conditions, we take $D=[0,1]$. We parameterize these coordinate functions by linear combinations of suitable basis functions. That way, we represent the closed curve $\mathbf{r}$ as

$$
\mathbf{r}(t)=\sum_{k=-\infty}^{\infty} \mathbf{c}[k] \varphi(M t-k),
$$

where $\varphi$ is a compactly supported generating function and $\left\{\mathbf{c}[k]=\left(c_{1}[k], c_{2}[k]\right)\right\}_{k \in \mathbb{Z}}$ is an $M$-periodic sequence of control points. Expressing $\mathbf{r}$ as in (1) allows us to take advantage of fast and stable interpolation algorithms [23]. The capability of $\mathbf{r}$ to adapt to detailed shapes is determined by the number of control points $M$ that determines the degrees of freedom in the model (1). Typically, larger $M$ leads to additional flexibility of the curve.

Under periodicity conditions, we can reduce the infinite summation in (1) to a finite one involving periodized basis functions as

$$
\begin{aligned}
\mathbf{r}(t) & =\sum_{k=0}^{M-1} \sum_{n=-\infty}^{\infty} \mathbf{c}[M n+k] \varphi(M(t-n)-k) \\
& =\sum_{k=0}^{M-1} \mathbf{c}[k] \underbrace{\sum_{n=-\infty}^{\infty} \varphi(M(t-n)-k)}_{\varphi_{M}(M t-k)},
\end{aligned}
$$

where $\varphi_{M}$ is the $M$-periodization of the basis function $\varphi$. An example of a curve described by (3) is shown in Figure 1. If $\varphi$ satisfies some mild conditions [24], then the defined parameterization is able to approximate any closed curve with arbitrary precision by using a sufficiently large number of vector coefficients. The function $\varphi$ determines multiple properties of the parametric model such as smoothness, reproduction of interesting shapes, or computational efficiency [25]. In practice, the particular choice of $\varphi$ is usually governed by two factors: computational complexity of the overall segmentation algorithm and capability of the snake to adopt specific shapes and to retain smoothness. B-spline functions have traditionally been used as basis functions due to the existence of efficient interpolation algorithms [26], their finite support, 
and their good approximation properties [27]. A typical design constraint inherited from the time when snakes were built out of polygons (or, equivalently, linear B-splines) is to minimize the overall curvature. Using the well-known variational properties of splines [28], one can show that the minimization of the curvature subject to interpolation constraints yields a cubicspline curve. However, traditional B-splines can only generate curves that can be expressed as piecewise polynomials. Recent research has lead the field to experiment with other basis functions that are more suited to particular applications [13], [29].

\section{B. Desirable Properties for the Basis Functions}

The three following conditions should be satisfied by our parametric snake model:

1) Unique Choice of Basis Functions and Stable Representation: We want our parametric curve to be defined in terms of the coefficients in such a way that unicity of representation is satisfied. Additionally, for computational purposes, the interpolation procedure must be numerically stable. Therefore, $\varphi$ has to satisfy the Riesz-basis condition, meaning that two constants $0<A \leq B<\infty$ have to exist, such that

$$
A\|\mathbf{c}\|_{\ell_{2}} \leq \sqrt{M}\|\mathbf{r}\|_{L_{2}} \leq B\|\mathbf{c}\|_{\ell_{2}}
$$

for all $\mathbf{c} \in \ell_{2}$. This inequality implies that

$$
\sum_{k=-\infty}^{\infty} \mathbf{c}[k] \varphi(M t-k)=0 \Rightarrow \mathbf{c}[k]=0 \forall k \in \mathbb{Z} .
$$

Thus, the basis functions are linearly independent and every function is uniquely specified by its coefficients. The upper inequality of (4) ensures the stability of the interpolation process [23]. A convenient way to verify whether a function $\varphi$ satisfies the Riesz-basis condition (4) is to express it in the Fourier domain [30] as

$$
A \leq \sum_{k=-\infty}^{\infty}|\hat{\varphi}(\omega+2 \pi k)|^{2} \leq B
$$

where $\hat{\varphi}(\omega)=\int_{\mathbb{R}} \varphi(x) \mathrm{e}^{-\mathrm{j} \omega x} \mathrm{~d} x$ denotes the Fourier transform of $\varphi$.

2) Affine Invariance: We want to represent shapes irrespectively of their position and orientation and, therefore, we would like our model to be invariant to affine transformations, which we formalize as

$$
\mathbf{A} \mathbf{r}(t)+\mathbf{b}=\sum_{k=-\infty}^{\infty}(\mathbf{A} \mathbf{c}[k]+\mathbf{b}) \varphi(M t-k),
$$

where $\mathbf{A}$ is a $(2 \times 2)$ matrix and $\mathbf{b}$ is a $2 \mathrm{D}$ vector. A more compact expression of affine transformations is obtained using homogeneous coordinates. We denote the homogeneous representation of a $2 \mathrm{D}$ column vector adding $h$ as a subscript. For instance, we write

$$
\mathbf{p}_{h}=\left(\begin{array}{c}
\mathbf{p} \\
1
\end{array}\right)
$$

With this particular notation, it is possible to represent both the linear transformation and the translation vector as a single matrix multiplication with the matrix

$$
\mathbf{H}=\left(\begin{array}{cc}
\mathbf{A} & \mathbf{b} \\
\mathbf{0} & 1
\end{array}\right) \text {. }
$$

Then, the condition (5) can be written in homogeneous coordinates as

$$
\mathbf{H r}_{h}(t)=\sum_{k=-\infty}^{\infty} \mathbf{H} \mathbf{c}_{h}[k] \varphi(M t-k)
$$

It follows that the affine invariance is ensured if and only if

$$
\forall t \in \mathbb{R}: \sum_{k=-\infty}^{\infty} \varphi(M t-k)=1 .
$$

In the literature, this constraint is often named the partition-of-unity condition [23] and is an intrinsic property of the basis function $\varphi$.

3) Linear Span of the Shape Space: The basis functions should be chosen so that they span a space that contains the curve to be segmented. Hence, they should be able to handle intrinsic properties of the desired shape, such as discontinuities or smoothness.

\section{EFFICIENT SHAPE PRIORS}

The aim of this section is to properly define the space that comprises all possible affine transformations of the reference curve, as well as the projector that maps any parametric curve onto this shape space.

\section{A. Notation}

In our framework, the prior knowledge about the shape is encoded in a parametric curve $\mathbf{r}^{\text {ref }}$ that takes the form of (1). It is referred to as the reference curve. The set of all affine transformations of the reference curve form the vector-space $\mathcal{S}_{\text {ref }}$, called the affine shape space. Finally, the orthogonal projection of any parametric shape onto the affine shape space is denoted by $\mathbf{r}^{\mathrm{p}}$.

\section{B. Parameterization of the Affine Shape Space}

Any curve in homogeneous coordinates within $\mathcal{S}_{\text {ref }}$ can be expressed as $\mathbf{H} \mathbf{r}_{h}^{\text {ref }}$, for some homogeneous $\mathbf{H}$ of the form (6). Since the reference curve is built as an expansion of the $M$ control points $\left\{\mathbf{c}^{\mathrm{ref}}[k]=\left(c_{1}^{\mathrm{ref}}[k], c_{2}^{\mathrm{ref}}[k]\right)\right\}_{k \in[0 \ldots M-1]}$ as in (3), the affine shape space is fully determined by these $M$ vector parameters. Moreover, since an affine transformation is uniquely determined by 6 scalar parameters, the space $\mathcal{S}_{\text {ref }}$ has dimension 6 .

\section{Shape Fitting Within the Affine Shape Space}

The best fit to the reference spline curve of a given curve $\mathbf{r}$ corresponds to its orthogonal projection $\mathbf{r}^{\mathrm{p}}$ onto $\mathcal{S}_{\text {ref }}$. Formally, the projected curve $\mathbf{r}^{\mathrm{p}}$ minimizes the mean-squared error (MSE), i.e.,

$$
\operatorname{MSE}=\int_{0}^{1}\left\|\mathbf{r}(t)-\mathbf{r}^{\mathrm{p}}(t)\right\|^{2} \mathrm{~d} t
$$


Note that the MSE can be rewritten using homogeneous coordinates as

$$
\operatorname{MSE}=\int_{0}^{1}\left\|\mathbf{r}_{h}(t)-\mathbf{r}_{h}^{\mathrm{p}}(t)\right\|^{2} \mathrm{~d} t .
$$

where

$$
\mathbf{r}_{h}^{\mathrm{p}}=\mathbf{H} \mathbf{r}_{h}^{\mathrm{ref}}
$$

and $\mathbf{H}$ is a $(3 \times 3)$ homogeneous affine matrix such as $(6)$.

We define the matrix representation of the set of homogeneous control points $\left\{\mathbf{c}_{h}[k]=\left(c_{1}[k], c_{2}[k], 1\right)\right\}_{k \in[0 \ldots M-1]}$ of a generic spline curve $\mathbf{r}_{h}$ as

$$
\begin{aligned}
\mathbf{C}_{h} & =\left(\begin{array}{c}
\mathbf{c}_{h}[0]^{\mathrm{T}} \\
\vdots \\
\mathbf{c}_{h}[M-1]^{\mathrm{T}}
\end{array}\right) \\
& =\left(\begin{array}{ccc}
c_{1}[0] & c_{2}[0] & 1 \\
\vdots & \vdots & \vdots \\
c_{1}[M-1] & c_{2}[M-1] & 1
\end{array}\right) .
\end{aligned}
$$

Analogously, $\mathbf{C}_{h}^{\text {ref }}$ and $\mathbf{C}_{h}^{\mathrm{p}}$ correspond to the matrix representation of the control points of the curves $\mathbf{r}_{h}^{\text {ref }}$ and $\mathbf{r}_{h}^{\mathrm{p}}$, respectively.

Theorem 1 provides an explicit expression to compute the control points $\mathbf{c}_{h}^{\mathrm{p}}$ of the projected curve $\mathbf{r}_{h}^{\mathrm{p}}$ as a linear mapping in $\mathbb{R}^{M}$. The analysis of this mapping reveals that the shape space is a 3D invariant subspace of $\mathbb{R}^{M}$.

Theorem 1: The matrix representation of the orthogonal projection in the $L_{2}([0,1])$ sense of a curve defined by $\mathbf{C}_{h}$ onto $\mathcal{S}_{\text {ref }}$ can be expressed by

$$
\mathbf{C}_{h}^{\mathrm{p}}=\mathbf{P}^{\mathrm{ref}} \mathbf{C}_{h},
$$

where $\mathbf{P}^{\text {ref }}$ is an $(M \times M)$ matrix given by

$$
\mathbf{P}^{\mathrm{ref}}=\mathbf{C}_{h}^{\mathrm{ref}}\left(\mathbf{C}_{h}^{\mathrm{ref}}{ }^{\mathrm{T}} \boldsymbol{\Phi} \mathbf{C}_{h}^{\mathrm{ref}}\right)^{-1} \mathbf{C}_{h}^{\mathrm{ref} \mathrm{T}} \boldsymbol{\Phi}
$$

$\boldsymbol{\Phi}$ is the autocorrelation matrix of the periodized basis functions, with

$$
\boldsymbol{\Phi}_{i, j}=\int_{0}^{1} \varphi_{M}(M t-i) \varphi_{M}(M t-j) \mathrm{d} t .
$$

Proof: We first expand (8) as

$$
\begin{aligned}
\mathrm{MSE}= & \int_{0}^{1}\left\|\mathbf{r}_{h}(t)-\mathbf{r}_{h}^{\mathrm{p}}(t)\right\|^{2} \mathrm{~d} t \\
= & \int_{0}^{1}\left(\mathbf{r}_{h}(t)-\mathbf{r}_{h}^{\mathrm{p}}(t)\right)^{\mathrm{T}}\left(\mathbf{r}_{h}(t)-\mathbf{r}_{h}^{\mathrm{p}}(t)\right) \mathrm{d} t \\
= & \int_{0}^{1}\left(\mathbf{r}_{h}(t)^{\mathrm{T}} \mathbf{r}_{h}(t)-2 \mathbf{r}_{h}(t)^{\mathrm{T}} \mathbf{r}_{h}^{\mathrm{p}}(t)\right. \\
& \left.+\mathbf{r}_{h}^{\mathrm{p}}(t)^{\mathrm{T}} \mathbf{r}_{h}^{\mathrm{p}}(t)\right) \mathrm{d} t .
\end{aligned}
$$

It follows that the minimizer of the MSE with respect to $\mathbf{H}$ also minimizes

$$
J=\int_{0}^{1}\left(-2 \mathbf{r}_{h}(t)^{\mathrm{T}} \mathbf{r}_{h}^{\mathrm{p}}(t)+\mathbf{r}_{h}^{\mathrm{p}}(t)^{\mathrm{T}} \mathbf{r}_{h}^{\mathrm{p}}(t)\right) \mathrm{d} t
$$

since $\mathbf{r}_{h}$ does not depend on $\mathbf{H}$.
Now, we expand each of the terms of the sum. By linearity, we obtain

$$
\begin{aligned}
& \mathbf{r}_{h}(t)^{\mathrm{T}} \mathbf{r}_{h}^{\mathrm{p}}(t) \\
& =\sum_{i=0}^{M-1} \sum_{j=0}^{M-1} \mathbf{c}_{h}[i]^{\mathrm{T}} \mathbf{c}_{h}^{\mathrm{p}}[j] \varphi_{M}(M t-i) \varphi_{M}(M t-j)
\end{aligned}
$$

and

$$
\begin{aligned}
& \mathbf{r}_{h}^{\mathrm{p}}(t)^{\mathrm{T}} \mathbf{r}_{h}^{\mathrm{p}}(t) \\
& =\sum_{i=0}^{M-1} \sum_{j=0}^{M-1} \mathbf{c}_{h}^{\mathrm{p}}[i]^{\mathrm{T}} \mathbf{c}_{h}^{\mathrm{p}}[j] \varphi_{M}(M t-i) \varphi_{M}(M t-j) .
\end{aligned}
$$

We obtain an explicit representation of $J$ in terms of the sequence of vector coefficients by substituting the above expression in (10). Then, we have

$$
J=\sum_{i=0}^{M-1} \sum_{j=0}^{M-1}\left(-2 \mathbf{c}_{h}[i]^{\mathrm{T}} \mathbf{c}_{h}^{\mathrm{p}}[j]+\mathbf{c}_{h}^{\mathrm{p}}[i]^{\mathrm{T}} \mathbf{c}_{h}^{\mathrm{p}}[j]\right) \boldsymbol{\Phi}_{i, j},
$$

where

$$
\boldsymbol{\Phi}_{i, j}=\int_{0}^{1} \varphi_{M}(M t-i) \varphi_{M}(M t-j) \mathrm{d} t
$$

are the samples of the autocorrelation of the periodized basis function. Finally, we can write $J$ using matrix notation as

$$
J=-2 \operatorname{tr}\left(\mathbf{C}_{h}^{\mathrm{T}} \boldsymbol{\Phi} \mathbf{C}_{h}^{\mathrm{p}}\right)+\operatorname{tr}\left(\mathbf{C}_{h}^{\mathrm{pT}} \boldsymbol{\Phi} \mathbf{C}_{h}^{\mathrm{p}}\right) .
$$

Equivalently, we can make explicit the dependency on the affine transformation by using the fact that the coefficients of the projected curve and the reference curve are related only by the affine transformation, $\mathbf{c}_{h}^{\mathrm{p}}=\mathbf{H} \mathbf{c}_{h}^{\text {ref }}$. Note that $\mathbf{c}_{h}^{\mathrm{p}}=\mathbf{H} \mathbf{c}_{h}^{\text {ref }}$ holds if and only if (9) holds. This can be shown by using the partition-of-unity property of $\varphi$, and the linearity of the model. In matrix notation, this amounts to substituting $\mathbf{C}_{h}^{\mathrm{p}}=\mathbf{C}_{h}^{\mathrm{ref}} \mathbf{H}^{\mathrm{T}}$ in (11). We obtain

$$
J=-2 \operatorname{tr}\left(\mathbf{C}_{h}^{\mathrm{T}} \boldsymbol{\Phi} \mathbf{C}_{h}^{\mathrm{ref}} \mathbf{H}^{\mathrm{T}}\right)+\operatorname{tr}\left(\mathbf{H} \mathbf{C}_{h}^{\mathrm{ref}}{ }^{\mathrm{T}} \boldsymbol{\Phi} \mathbf{C}_{h}^{\mathrm{ref}} \mathbf{H}^{\mathrm{T}}\right) .
$$

Now, to obtain the optimal affine transformation in $\mathbf{H}$, we equate the gradient of $J$ with respect to $\mathbf{A}$ and $\mathbf{b}$ to zero. It can be shown that the solution is equivalent to the one obtained by computing the derivative with respect to the free components of $\mathbf{H}$ constrained to the knowledge of the last row. We define $\frac{\partial^{\prime} J}{\partial \mathbf{H}}$ as the derivative of $J$ with respect to the free components of $\mathbf{H}$. More precisely,

$$
\frac{\partial^{\prime} J}{\partial \mathbf{H}}=\left(\begin{array}{ccc}
\frac{\partial J}{\partial a_{11}} & \frac{\partial J}{\partial a_{21}} & 0 \\
\frac{\partial J}{\partial a_{12}} & \frac{\partial J}{\partial a_{22}} & 0 \\
\frac{\partial J}{\partial b_{1}} & \frac{\partial J}{\partial b_{2}} & 0
\end{array}\right),
$$

where $\mathbf{H}$ is a generic affine matrix as given by (6) whose explicit expression is

$$
\mathbf{H}=\left(\begin{array}{ccc}
a_{11} & a_{12} & b_{1} \\
a_{21} & a_{22} & b_{2} \\
0 & 0 & 1
\end{array}\right)
$$


Then, the optimality condition becomes

$$
\begin{aligned}
\frac{\partial^{\prime} J}{\partial \mathbf{H}}= & -2\left(\mathbf{C}_{h}^{\mathrm{T}} \boldsymbol{\Phi}^{\mathrm{T}} \mathbf{C}_{h}^{\mathrm{ref}}\right)^{\mathrm{T}} \\
& +\mathbf{C}_{h}^{\mathrm{ref}}{ }^{\mathrm{T}} \boldsymbol{\Phi} \mathbf{C}_{h}^{\mathrm{ref}} \mathbf{H}^{\mathrm{T}}+\mathbf{C}_{h}^{\mathrm{ref}} \boldsymbol{\Phi}^{\mathrm{T}} \mathbf{C}_{h}^{\mathrm{ref}} \mathbf{H}^{\mathrm{T}} \\
= & \mathbf{0} .
\end{aligned}
$$

We regroup the components, and express the equality as

$$
-2 \mathbf{C}_{h}^{\mathrm{ref}^{\mathrm{T}}} \boldsymbol{\Phi} \mathbf{C}_{h}+\mathbf{C}_{h}^{\mathrm{ref}^{\mathrm{T}}}\left(\boldsymbol{\Phi}+\boldsymbol{\Phi}^{\mathrm{T}}\right) \mathbf{C}_{h}^{\mathrm{ref}} \mathbf{H}^{\mathrm{T}}=\mathbf{0}
$$

Finally, since $\boldsymbol{\Phi}$ is a positive-definite symmetric matrix, the optimal affine transformation is

$$
\mathbf{H}=\mathbf{C}_{h}^{\mathrm{T}} \boldsymbol{\Phi} \mathbf{C}_{h}^{\mathrm{ref}}\left(\mathbf{C}_{h}^{\mathrm{ref}} \boldsymbol{\Phi} \mathbf{C}_{h}^{\mathrm{ref}}\right)^{-1}
$$

The matrix representation of the control points of the projected curve is obtained as

$$
\mathbf{C}_{h}^{\mathrm{p}}=\mathbf{C}_{h}^{\mathrm{ref}}\left(\mathbf{C}_{h}^{\mathrm{ref}}{ }^{\mathrm{T}} \boldsymbol{\Phi} \mathbf{C}_{h}^{\mathrm{ref}}\right)^{-1} \mathbf{C}_{h}^{\mathrm{ref}} \boldsymbol{\Phi} \mathbf{C}_{h}
$$

By identification from (12), we finally obtain that

$$
\mathbf{P}^{\mathrm{ref}}=\mathbf{C}_{h}^{\mathrm{ref}}\left(\mathbf{C}_{h}^{\mathrm{ref}}{ }^{\mathrm{T}} \boldsymbol{\Phi} \mathbf{C}_{h}^{\mathrm{ref}}\right)^{-1} \mathbf{C}_{h}^{\mathrm{ref}^{\mathrm{T}}} \boldsymbol{\Phi} .
$$

Note that $\mathbf{P}^{\text {ref }}$ can be precomputed since it only depends on the control points of the reference curve. This allows for a fast implementation of the projection operator. The implementation details are presented in Section VI-A.

The properties of the matrix $\mathbf{P}^{\text {ref }}$ are summarized as:

1) idempotence $\left(\mathbf{P}^{\mathrm{ref}^{2}}=\mathbf{P}^{\mathrm{ref}}\right)$;

2) rank 3, as long as the spline coefficients are not aligned;

3 ) the non-zero eigenvalues are equal to one and the corresponding eigenvectors are $\left(c_{1}^{\mathrm{ref}}[0], \ldots, c_{1}^{\mathrm{ref}}[M-1]\right)^{\mathrm{T}}$, $\left(c_{2}^{\mathrm{ref}}[0], \ldots, c_{2}^{\mathrm{ref}}[M-1]\right)^{\mathrm{T}}$, and $(1, \ldots, 1)^{\mathrm{T}}$.

These properties show that $\mathbf{P}^{\text {ref }}$ is an oblique projector from $\mathbb{R}^{M}$ to a 3D-invariant subspace of $\mathbb{R}^{M}$ defined by the vectors $\left(c_{1}^{\mathrm{ref}}[0], \ldots, c_{1}^{\mathrm{ref}}[M-1]\right), \quad\left(c_{2}^{\mathrm{ref}}[0], \ldots\right.$, $\left.c_{2}^{\text {ref }}[M-1]\right)$, and $(1, \ldots, 1)$. However, the corresponding projection of the curve onto $\mathcal{S}_{\text {ref }}$ in the $L_{2}$ sense is orthogonal since it minimizes the MSE in (7).

\section{Active Contours With Prior Shapes}

The evolution of the snake is formulated as a minimization problem; the associated cost function is usually denoted as snake energy. The snake energy, as initially introduced by Kass et al. [3] is a linear combination of three terms:

- the image energy $E_{\mathrm{image}}$ is responsible for guiding the snake towards the boundary of interest;

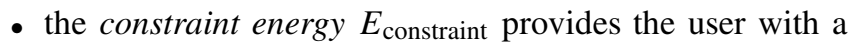
way to interact with the snake;

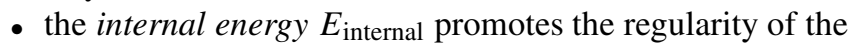
snake curve.

The overall snake energy is then given by

$$
E_{\text {snake }}(\Omega)=E_{\text {image }}(\Omega)+E_{\text {internal }}(\Omega)+E_{\text {constraint }}(\Omega),
$$

where $\Omega$ stands for the snake representation through parameters (e.g., control points). The snake optimization consists in finding

$$
\Omega^{*}=\underset{\Omega}{\arg \min } E_{\text {snake }}(\Omega) .
$$

The choice of each term is crucial because it has a direct impact on the quality of segmentation. It usually depends on the application and imaging modality. There is a vast amount of literature on designing such energies [7], [8], [31]-[33]. For the image energy, there are many strategies which can broadly be categorized in two families: 1) schemes, which use gradient information to detect contours (a.k.a. edge-based methods) and 2) methods, which use statistical information to distinguish different regions (a.k.a. region-based methods). In our model, we use a formulation where the image energy is a convex combination of an edge-based term and a region-based term [11], [13], i.e., $E_{\text {image }}=\alpha E_{\text {edge }}+(1-\alpha) E_{\text {region. Note }}$ that, for $\alpha=0$, the image energy reduces to a regionbased scheme, and, for $\alpha=1$, the image energy reduces to a edge-based scheme. We use the internal energy to encode the prior shape information by constructing a term that favors convergence of the snake towards a shape that is an affine transformation of a given reference shape. The exact expression is given in Section IV-A. We obviate the constraint energy in the formulation. In return, we accommodate the user interaction as a hard constraint by leaving selected coefficients out of the optimization routine. Finally, our overall snake energy is reduced to

$$
E_{\text {snake }}=\underbrace{\alpha E_{\text {edge }}+(1-\alpha) E_{\text {region }}}_{E_{\text {image }}}+E_{\text {internal }} .
$$

We now describe how to incorporate the information from the affine shape space introduced in Section III-C.

\section{A. Internal Energy With Shape Priors}

We define a new energy that encourages the snake to stay close to the shape space $\mathcal{S}_{\text {ref }}$. This energy penalizes shapes that are not affine transformations of the reference curve $\mathbf{r}^{\text {ref }}$. This is achieved by constructing a quadratic functional that achieves its minimum when the curve belongs to $\mathcal{S}_{\text {ref }}$, which represents the closest fit with respect to the reference shape. To that end, we define

$$
E_{\text {internal }}=\beta\left\|\left(\mathbf{I d}-\mathbf{P}^{\mathrm{ref}}\right) \mathbf{C}_{h}\right\|_{\mathrm{F}}^{2},
$$

where $\beta$ is a weight, $\mathbf{C}_{h}$ is the matrix form of the curve, $\mathbf{P}^{\text {ref }}$ is the projector onto $\mathcal{S}_{\text {ref }}$, and $\|\cdot\|_{\mathrm{F}}$ is the Frobenius norm. Note that the internal energy (15) is not equal to the MSE (7) in general. However, both expressions share the same minimizer (i.e., the coefficients of the optimal curve are the same) due to Theorem 1. The factor $\beta$ determines the trade-off between the adhesion to the boundaries within the image and regularization towards the prior shape. The minimization of (15) ensures the convergence to a shape that is an affine transformation of the reference shape; smoothness and regularity will therefore be preserved. 


\section{B. Continuous-Domain vs. Discrete Shape Priors}

The proposed prior shape model can also be transposed into the discrete setting using the conventional discrete shape-space formalism [1]. Using standard results from approximation theory, it can be shown that any curve in the plane (e.g., a pixel-based curve) can be approximated by a splinebased model [24] with an arbitrary precision. More precisely, the averaged minimum mean-squared approximation error along all possible parameterization shifts between both curves converges to zero at $\mathcal{O}\left(N^{-L}\right)$, where $N$ is the number of control points of the curve and $L$ is a strictly positive factor that depends exclusively on the generating function $\varphi$.

Let us consider a pixel-based curve obtained by uniformly sampling the parameter space of a continuous curve. We obtain the collection of points $\{\mathbf{r}(n / N)\}_{n=0 \ldots N-1}$. Such pointwise representation converges to the continuous curve when $N \rightarrow \infty$ as long as the curve contains no discontinuities [24]. In this situation, the continuous mean-squared error in (7) is equivalent to the classic discrete one as long as $N$ is large enough. Formally, we have

$$
\lim _{N \rightarrow \infty} \frac{1}{N} \sum_{n=0}^{N-1}\left\|\mathbf{r}\left(\frac{n}{N}\right)-\mathbf{r}^{\mathrm{p}}\left(\frac{n}{N}\right)\right\|^{2}=\int_{0}^{1}\left\|\mathbf{r}(t)-\mathbf{r}^{\mathrm{p}}(t)\right\|^{2} \mathrm{~d} t .
$$

In this discrete setting, an equivalent projection operation would be defined by an $N \times N$ matrix instead of the $M \times M$ matrix in the continuous case (see Theorem 1). Spline-snakes are usually built with a small number of control points. For instance, in the experimental validation of Section $\mathrm{V}$, we use a number of control points $M$ ranging within the interval $[4,9]$. In the presented discrete setting, $N$ is usually chosen to match the spatial resolution of the image, that means that the average distance in the plane between consecutive samples is close to one-pixel unit. Equivalent point-based contours to the ones shown in Section $\mathrm{V}$ achieving the same spatial resolution would contain $N \approx 1000$ discrete points (value depending on image resolution). Since $N \gg M$ and the continuous and discrete minimization criteria are equivalent thanks to (16), we see that the presented model provides an equivalent shape prior to the snake at a much lower computational complexity.

\section{Optimization of the Snake}

The optimization is carried out efficiently by a Powell-like line-search method [34]. The algorithm proceeds as follows: First, a promising direction within the parameter space is chosen depending on the partial derivatives of the energy. Second, a 1D minimization is performed along the selected direction. Finally, a new direction is chosen using the partial derivatives of the energy function once more, while enforcing conjugation properties. This scheme is repeated until convergence, with occasional conjugation reset. This method requires either the partial derivatives of the energy function with respect to the parameters or, in our case, with respect to the control points. A detailed derivation of the gradient of the energy described by (13) can be found in [11]. (a)

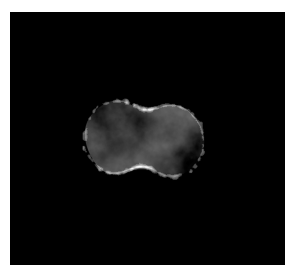

(b)

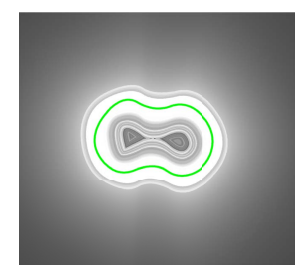

(c)

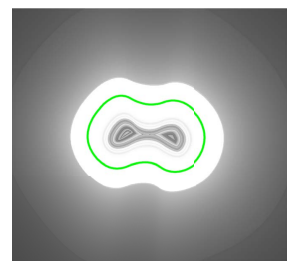

Fig. 2. Study of basin of attraction for simulated data. (a) Synthetic image of a mitotic cell model. (b) Basin of attraction of the snake with $\beta=0$ with the ground truth overlaid. (c) Basin of attraction of the snake with $\beta \neq 0$ with the ground truth overlaid. The gray values of each pixel location in (b) and (c) correspond to the averaged Jaccard indices of the snakes going through each pixel location.

The contribution of the proposed shape-based internal energy can be extracted from the two first columns of

$$
\begin{gathered}
\left(\begin{array}{ccc}
\frac{\partial E_{\text {internal }}}{\partial c_{1}[0]} & \frac{\partial E_{\text {internal }}}{\partial c_{2}[0]} & 0 \\
\vdots & \vdots & \vdots \\
\frac{\partial E_{\text {internal }}}{\partial c_{1}[M-1]} & \frac{\partial E_{\text {internal }}}{\partial c_{2}[M-1]} & 0
\end{array}\right) \\
=2 \beta\left(\mathbf{I d}-\mathbf{P}^{\text {ref }}\right)^{\mathrm{T}}\left(\mathbf{I d}-\mathbf{P}^{\text {ref }}\right) \mathbf{C}_{h} .
\end{gathered}
$$

\section{VALIDATION AND EXPERIMENTS}

In this section, we evaluate the effect of the presented energy term in different typical situations encountered in biomedical imaging. First, we experimentally illustrate the snake's sensitivity to initial conditions. Then, we analyze the robustness to noise of the model by segmenting simulated images containing different levels of noise. Next, we show the effect of the coefficient $\beta$ when segmenting in low-contrast situations. We conclude by analyzing the performance on the method on real biological data. In the following experiments as well as in the proposed software, the contour is built using the ellipse-reproducing basis functions $\varphi$ described in [13].

\section{A. Dependence on Initial Conditions}

Active contour models are known to be strongly dependent on initial conditions. Sensitivity to the location and shape of the initial contour is difficult to study thoroughly, and quantitative evaluation of this aspect is therefore rarely observed analytically. In order to address the question of the dependence of snake convergence on initial conditions, we have experimentally computed the basin of attraction of a snake optimized using the proposed energy term.

We have created an 8-bit synthetic image of a typical mitotic cell in fluorescence microscopy (see Figure 2a). To mimic realistic conditions, the outline of our synthetic dividing cell contains holes and intensity variations. Moreover, the cell's inside simulates inhomogeneous background in the fluorescence signal. The ground truth was constructed by manually placing a spline curve with 9 control points perfectly matching the edge of the cell. We initialized 50 different snakes at random locations constructed from random perturbations of the spline curve used as ground truth. The perturbed contours were automatically optimized with $\beta=0$ (no shape prior energy) and $\alpha=1$ in (14) (i.e., no region-based term) using 
TABLE I

AREA OF BASIN OF ATTRACTION YIELDING

Perfect Segmentation $\left(J_{I}>0.9\right)$

\begin{tabular}{cc}
\hline \hline$\beta$ & area \\
\hline 0 & 147716 \\
10 & 279800 \\
\hline \hline
\end{tabular}

an edge-based term relying on a popular ridge-estimator based on the eigen-decomposition of the smoothed Hessian matrix of the image [35]-[37]. Then, the experiment was repeated with $\beta=10$ using a shape prior derived from the ground truth. The value of $\beta$ was chosen experimentally.

To evaluate the sensitivity to initial conditions, the Jaccard index, $J_{I}=\frac{|A \cap B|}{|A \cup B|}$, where $A$ and $B$ are two non-empty sets, was computed between all snakes and the ground truth. A high value of $J_{I}$ reflects the fact that the snake managed to find its way back to the optimal configuration. We show in Figure 2b and Figure 2c the averaged values of the $J_{I}$ for the 50 repetitions. The gray values of each pixel location in the images correspond to the averaged Jaccard indices of the snakes that go through that particular pixel location. In Table I, we show the size of the basin of attraction as defined by the pixel locations with $J_{I}>0.9$. One can observe that using the prior shape energy term results in a two-fold increase in the area of the basin of convergence as long as the prior shape is well matched to the object to segment.

\section{B. Efficiency and Robustness to Noise}

In order to evaluate the proposed energy term with respect to the robustness to noise, we used the same test image, ground truth and energy terms as in Section V-A. We generated 10 noisy realizations for several noise levels $(\sigma=40,70,80$, and 90), where $\sigma$ corresponds to its standard deviation by adding Gaussian white noise to the original synthetic image. The resulting images have signal-to-noise ratios of $-6.6 \mathrm{~dB},-11.4 \mathrm{~dB},-12.54 \mathrm{~dB}$, and $-13.46 \mathrm{~dB}$. Through all experiments, the snake was initialized from the position shown in Figure 3 (top left), which corresponds to a Jaccard index of $J_{I}=0.59$. Performance measures of the segmentation algorithm are given as Jaccard indices (see Table II), and visual representation of the results are shown in Figure 3. As in SectionV-A, a value of $\beta=10$ was used for this experiment. One can observe that the influence of the shape prior increases the robustness to noise of the algorithm. In particular, the performance of the algorithm without prior degrades as the noise power increases whereas the version with the shape prior converges to the same solution with $J_{I}=0.96$. Moreover, the standard deviation of the $J_{I}$ remains stable for the experiments with shape prior while it increases substantially in the experiments without any prior.

\section{Overall Contribution of Prior Shape Energy}

The coefficient $\beta$ in (15) can be interpreted as a balance factor between the contribution of the data term and the influence of the shape prior. It can be arbitrarily large, and for very high values it will force the snake to lie within the affine shape space given by the prior. This point is important since

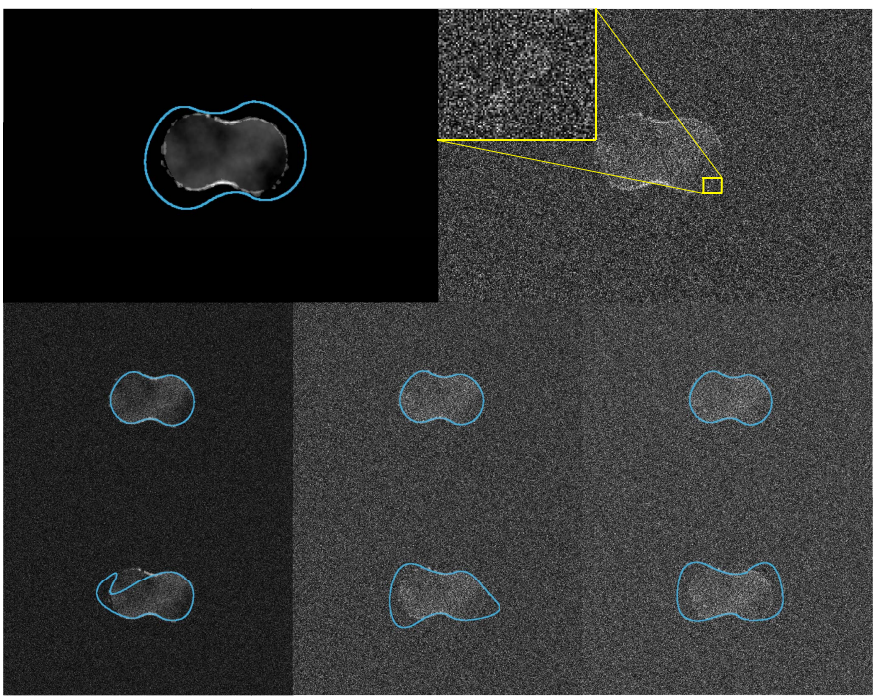

Fig. 3. Segmentation of synthetic data in the presence of noise. Top row: initialization (left) and noisy image (right). A close up of a boundary region between the artificial cell and the background is shown. Central row: segmentation with shape prior in noisy conditions: the synthetic image has been corrupted by adding AGWN with standard deviations of 40 (left), 80 (center), and 90 (right). Bottom row: same experiment as center row, but without using a shape prior.

TABLE II

JACCARD INDICES FOR SEGMENTATION OF (NOISY) DATA

\begin{tabular}{ccc}
\hline \hline SNR [dB] (stdd) & shape prior & no prior \\
\hline$\infty$ (noiseless) & 0.96 & 0.96 \\
$-6.60(40)$ & $0.96 \pm 0.0030$ & $0.74 \pm 0.0061$ (fail) \\
$-11.4(70)$ & $0.96 \pm 0.0027$ & $0.76 \pm 0.0164$ (fail) \\
$-12.54(80)$ & $0.96 \pm 0.0023$ & $0.72 \pm 0.0259$ (fail) \\
$-13.46(90)$ & $0.96 \pm 0.0019$ & $0.67 \pm 0.0672$ (fail) \\
\hline
\end{tabular}

an extremely large value of $\beta$ would make the system obviate the image energy completely and converge to the closest shape within the span of the shape-space in terms of (7) regardless of the image. However, this configuration could be very different in terms of $J_{I}$. Therefore, the minimization of (7) does not always imply an optimal value for $J_{I}$.

We tested the influence of $\beta$ by segmenting the noise-free test image described in the previous section and that is illustrated both on top left of Figure 3 and on Figure 2a. We manually initialized our snake such that the overlap of this initialization and the ground truth corresponds to a Jaccard index of $J_{I}=0.52$. Figure 4 shows quantitatively how increasing the influence of the shape prior improves the segmentation. When a certain value of $\beta$ is reached $\left(\beta \approx 10^{6}\right.$ in our experiment), the shape prior energy dominates the system and the snake does not converge to the shape of interest anymore. We see that when $J_{I} \approx 10^{7}$, the value of $J_{I}$ is similar to the initial value before optimization, i.e., $J_{I}=0.52$. This is due to numerical precision in the iterative optimization process since the gradient of the image energy is nearly zero in comparison to the value of the gradient of the prior shape energy.

\section{Segmentation of Drosophila Flies}

In this experiment, we have tested the prior energy term on real time-lapse sequences of Drosophila flies taken with high-frame-rate cameras. The fruit fly, Drosophila, is a 


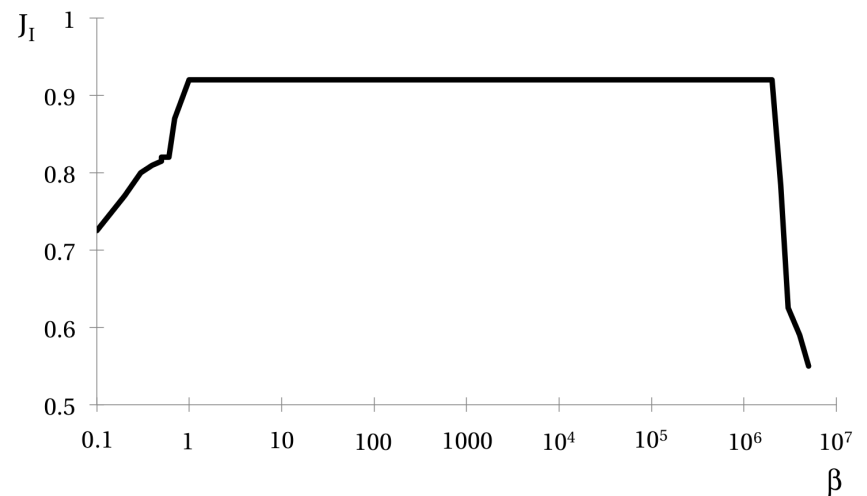

Fig. 4. Effect of the balance parameter $\beta$ when segmenting the simulated data displayed both on top left of Figure 3 and on Figure $2 \mathrm{a}$. The effect of the shape prior energy increases with $\beta$. In the limit case when $\beta=0$, the snake is completely driven by the image energy. Conversely, when $\beta$ is large enough, the system is dominated by the shape prior energy.

typical model organism for the study of voluntary locomotion. Its nervous system is simple and can be easily manipulated for experimental purpose. Sequences of images featuring flies walking inside a confined environment under different experimental conditions are usually the data of choice for such a study. The wandering behavior of Drosophila must then be quantified with high precision in order to understand the underlying locomotion mechanism of the fly [38].

In order to segment the fly, we used the edge-based term mentioned in Section V-A and a region-based term that discriminates an object from its background by building a shell around the snake and maximizing the contrast between the intensity of the image averaged within the snake curve and the intensity of the image averaged over the elliptical shell [9], [13]. As reference shape, we used the symmetric fly model from our library with 18 control points as depicted in Figure $8 \mathrm{~g}$. The effect of optimizing the snake with or without the shape prior is shown in Figure 5. In particular, in cases where the shape prior is not used, the snake converges to a solution where the abdomen is well captured but the head suffers from an extremely coarse segmentation. This is due to the fact that nearby leg segments create spurious edges for the edge-based energy term, and the uniformity of brightness in that region image does not allow the region-based term to properly discriminate all features of the head. However, when adding the shape-based component, the snake is able to catch the head of the fly similarly well regardless of the image energy used.

\section{E. Segmentation of Yeast Cells}

We have applied the proposed energy term to segment the Schizosaccharomyces pombe (a.k.a. fission yeast) [10]. Two different data sets have been used: one to train a shape prior and a second for the validation. The training set is shown in Figure 6a. The cells were manually segmented by a biologist and subsequently aligned in order to compute a statistical mean shape [39]. In order to segment the yeast cells, we used the image energy term mentioned in Section V-D with $\alpha=0.5$ and the mean prior shape learned from the data with $\beta=0.5$. The initialization of each snake was performed manually (a)

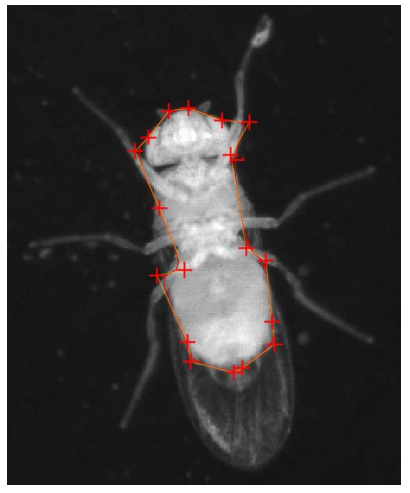

(c)

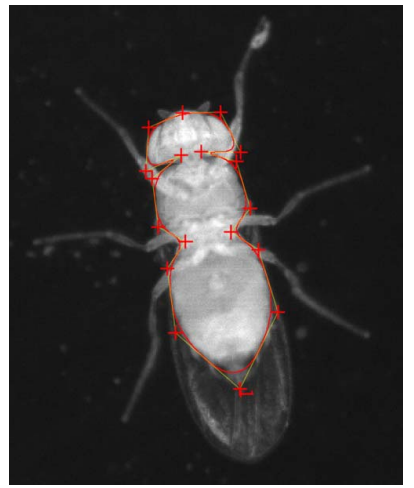

(b)

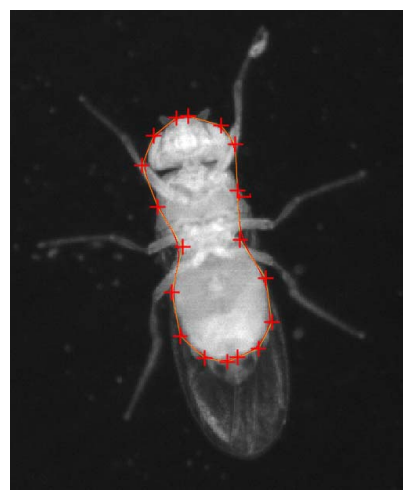

(d)

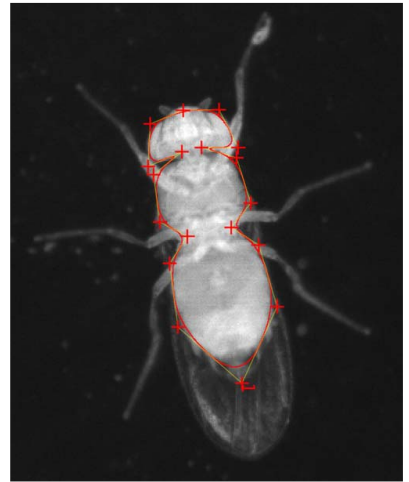

Fig. 5. Qualitative assessment of the influence of the shape prior term while segmenting a Drosophila fly. (a) Edge-based term only (i.e., $\alpha=1$ and $\beta=0$ ). (b) Region-based term only (i.e., $\alpha=0$ and $\beta=0$ ). (c) Edge-based term and shape-based energy (i.e., $\alpha=1$ and $\beta>0$ ). (d) Region-based term and shape-based energy (i.e., $\alpha=0$ and $\beta>0$ ). The ' + ' elements represent the control points of the snake, which can still be fine-tuned manually to increase the accuracy of the segmentation. Image courtesy of the Laboratory of Intelligent Systems, EPFL, Switzerland.

and is shown in Figure 6b. All snakes were composed of 8 control points. Finally, the results of the segmentation of the validation set are shown in Figure 6c. In order to provide quantitative performance results, the same validation set was also segmented by a biologist, thus providing ground truth. Several performance measures, in terms of Jaccard indices, between the results obtained by our algorithm and the ground truth are shown in Table III. We see that the results are in agreement with the biologist and consistent with the Jaccard indices obtained with simulated data.

\section{F. Computational Aspects}

The cost of evaluation of the prior shape energy per iteration boils down to a simple low-dimensional matrix-vector operation, where the matrix is of size $M \times M$. We verified experimentally that this cost is negligible with respect to the evaluation of the image energy. For this purpose we measured the time to run 1000 iterations with and without shape prior energy. We repeated the experiment 10 times. In both cases we obtained $76 \pm 2$ milliseconds, which shows the efficiency of the proposed energy term. The experiment was carried out on a $3.5 \mathrm{GHz}$ processor with $32 \mathrm{~GB}$ RAM.

In general, the computation of the image energy dominates the optimization of the snake. In particular, the complexity 
(a)

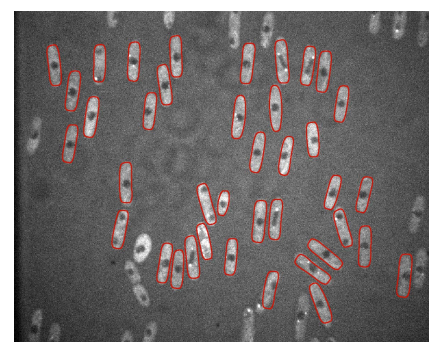

(b)

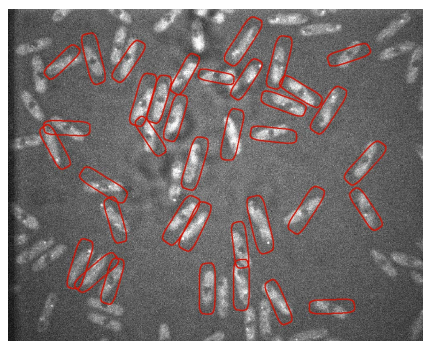

(c)

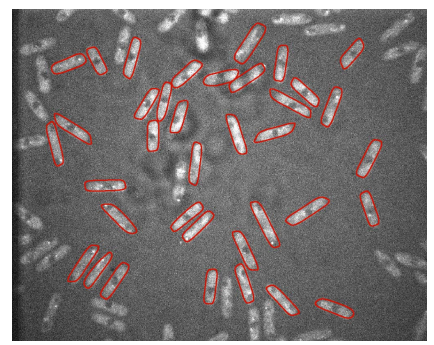

Fig. 6. Segmentation of the $S$. pombe. (a) Training set segmented manually by an expert. (b) Initialization of multiple 8-points snakes on the validation set using the learned prior shape from the training data. (c) Segmentation of the yeast cells using the shape prior. Image courtesy of the Simanis-lab, EPFL, Switzerland.

TABLE III

JACCARD INDICES FOR SEGMENTATION OF 37 YEAST CELLS

\begin{tabular}{cc}
\hline \hline measure & $J_{I}$ \\
\hline mean & 0.85 \\
std & 0.06 \\
min & 0.72 \\
max & 0.94 \\
\hline
\end{tabular}

of region-based terms is an order of magnitude higher than their edge-based counterparts. Most region-based terms can be expressed as surface integrals of an integrand over the domain enclosed by the curve [11]. An efficient way to implement the computation of the energy function is by using pre-integrated images [11], [13]. By Green's theorem, one can rewrite the surface integral as the line integrals over pre-integrated images. The use of Green's theorem to rewrite the surface integrals as line integrals dramatically reduces the computational load. This can only be achieved accurately if the curve is defined continuously.

Despite the fact that we are assuming a continuously defined model for our functions, in a real-world implementation we only have at our disposal a sampled version of the function we want to pre-integrate. To solve this inconsistency, we store the values of the pre-integrated images at integer locations in auxiliary image arrays. Then, the energies can be obtained using a bilinear interpolation of the sampled values in the auxiliary images. Details for particular implementations of this scheme can be found in [13] and [40].

\section{SOFTWARE}

\section{A. Implementation}

In order to ensure user-friendliness of the described algorithm, we implemented the framework in a modular way. The three different modules are: i) a dictionary containing (a)

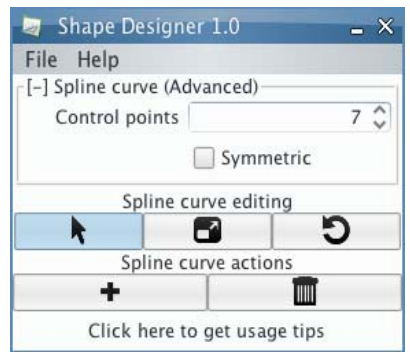

Fig. 7. Shape designer. (a) The GUI, and (b) an illustration of the use of the shape designer to segment a brain ventricle.

the shape library, ii) a routine to design and store custom spline shapes, and iii) the implementation of the segmentation algorithm. They are provided as freely available independent open-source plug-ins. The entire framework runs on Icy, an open-community Java-based platform for bio-image informatics. ${ }^{1}$ It can also be installed on tablets capable of running Java. In the following, we describe each of the modules and how they can be combined.

\section{B. Shape Designer for Icy}

The "Shape Designer" is an independent plug-in that enables the design of custom shapes based on splines. The idea is to let the user define shapes as refined as desired, without requiring any knowledge about the underlying mathematical properties of the curves. In Figure 7a, the GUI of the plug-in is shown. It can be used to draw the control polygon of a new spline snake to match a contour of interest in an image with a few mouse clicks. The number of control points can be specified by the user in order to delineate objects with arbitrary precision. The plug-in includes additional interactive functionalities to improve and facilitate manipulations, such as dilation and rotation of a shape with respect to its centroid, translation, and creation of symmetric shapes. In Figure 7b, the boundary of the ventricle of a brain on a slice of an MRI image has been segmented with the shape designer. In this example, $M=7$ control points have been used. The shapes that are designed with the shape designer can be saved in XML format to be reloaded to the shape library.

\section{Shape Library}

The shape library stores the shape priors that are used for the segmentation process. New shapes can be loaded as XML files to be used as custom shape priors. We list in Table IV some of the shapes that are provided by default. The letter in brackets indicates the corresponding shape shown in Figure 8 while $M$ is the number of control points used to generate the curve. To generate the shapes, we use the previously presented parametric spline snakes [13], [40] that perfectly reproduce ellipses, while at the same time being capable of approximating any planar closed curve. These basis functions have minimal support, which makes the optimization process

\footnotetext{
${ }^{1}$ http://icy.bioimageanalysis.org/
} 
(a)

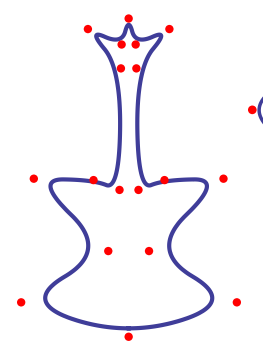

(b)

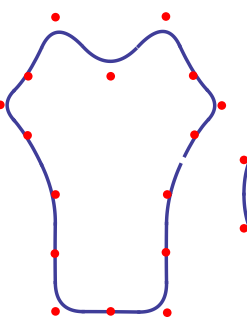

(c)

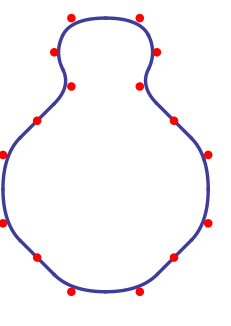

(d)

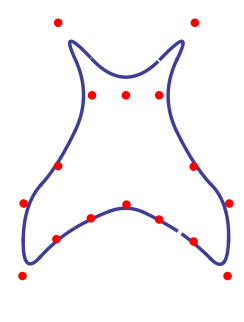

(e)

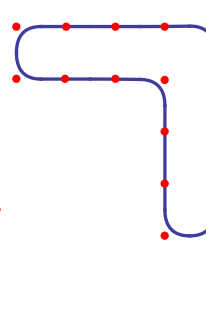

(f)

$(\mathrm{g})$

(h)

Fig. 8. Shape library, with some of the shapes available by default. The red dots indicate the location of the control points. (a) Guitar. (b) Femur bone. (c) Snow man. (d) Brain white matter. (e) Boomerang. (f) Corpus callosum. (g) Fly body. (h) Shamrock.

TABLE IV

Shapes Available in the Shape Library

\begin{tabular}{rr}
\hline \hline shape & $M$ \\
\hline guitar (a) & 18 \\
femur bone (b) & 16 \\
snow man (c) & 16 \\
brain white matter (d) & 16 \\
boomerang (e) & 16 \\
corpus callosum (f) & 16 \\
fly body (g) & 18 \\
shamrock (h) & 16 \\
\hline
\end{tabular}

\begin{tabular}{|c|c|c|c|}
\hline \multicolumn{3}{|c|}{ Active Celis 2.0} & $-x$ \\
\hline \multirow{2}{*}{\multicolumn{4}{|c|}{$\begin{array}{l}\text { File Analyze Help } \\
{[+] \text { Image (Basics) }}\end{array}$}} \\
\hline & & \multicolumn{2}{|c|}{ Image } \\
\hline \multicolumn{4}{|c|}{ [-] Snake (Advanced) } \\
\hline \multirow{2}{*}{\multicolumn{2}{|c|}{$\begin{array}{r}\text { Target brightness } \\
\text { Control points }\end{array}$}} & Dark & $v$ \\
\hline & & & $3 \hat{\imath}$ \\
\hline \multicolumn{2}{|c|}{ Energy type } & Region & $v$ \\
\hline \multicolumn{2}{|r|}{ Alpha } & & $\hat{\imath}$ \\
\hline \multicolumn{2}{|c|}{ Prior shape } & None & $v$ \\
\hline \multicolumn{2}{|r|}{ Beta } & Corpus callosum & $\wedge$ \\
\hline \multirow{3}{*}{\multicolumn{2}{|c|}{ Max iterations }} & Cross & \\
\hline & & Electric guitar & \\
\hline & & Femur & \\
\hline \multicolumn{2}{|r|}{ Snak } & Fly & \\
\hline+ & t & Mouse organ & \\
\hline \multicolumn{2}{|r|}{ Snak } & $\begin{array}{l}\text { Semicircle } \\
\text { Square }\end{array}$ & $\checkmark$ \\
\hline & & W & $\square$ \\
\hline \multicolumn{4}{|c|}{ Click here to get usage tips } \\
\hline
\end{tabular}

Fig. 9. Integration of the "shape designer" in the "active cells" plug-in.

more efficient [25]. After the shapes have been loaded, they can be chosen

\section{Integration With Active Cells}

The shape library is integrated in the main GUI of the "Active Cells" plug-in (Figure 9). Through it, the user can manipulate snakes and images. Different combinations of snake energies can be chosen, such as contour or region energies, and the relative weight can be easily adjusted. The use of shape priors is implemented by means of a supplementary energy function whose weight can also be adjusted. This facilitates user interaction and the results can be optimized online and in an intuitive way.

\section{CONCLUSION}

We have presented and derived a new energy term to efficiently include shape priors for the segmentation with 2D spline snakes. It consists of an orthogonal projector of an arbitrary shape onto the space that spans all the affine transformations of a given shape prior. The specificity of our approach is that the shape is represented by a parametric curve as opposed to a discrete point cloud. We have shown that the proposed projector is optimal in the $L_{2}$ sense. We gave an explicit expression of the corresponding energy term and of its gradient in order to use it in a conventional optimization scheme. The proposed algorithm has been implemented as a framework that combines the energy describing the shape prior together with traditional energy terms such as edge- or regionbased energies. A plug-in called "shape designer" has also been developed. It allows one to design custom spline shapes with a few mouse clicks, store, and finally reuse them in a shape library. The complete framework is implemented as a collection of user-friendly and interactive plug-ins. It is freely available through Icy. ${ }^{2}$ It has been successfully tested on real biological segmentation problems such as detecting fly bodies of Drosophila and yeast cells in microscopic images.

\section{REFERENCES}

[1] I. Dryden and K. Mardia, Statistical Shape Analysis. New York, NY, USA: Wiley, 1998.

[2] F. Precioso and M. Barlaud, "B-spline active contour with handling of topology changes for fast video segmentation," EURASIP J. Appl. Signal Process., vol. 2002, no. 1, pp. 555-560, Jan. 2002.

[3] M. Kass, A. Witkin, and D. Terzopoulos, "Snakes: Active contour models," Int. J. Comput. Vis., vol. 1, no. 4, pp. 321-331, Jan. 1987.

[4] A. Blake and M. Isard, Active Contours: The Application of Techniques From Graphics, Vision, Control Theory and Statistics to Visual Tracking of Shapes in Motion, 1st ed. New York, NY, USA: Springer-Verlag, 1998.

[5] R. Delgado-Gonzalo, V. Uhlmann, D. Schmitter, and M. Unser, "Snakes on a plane: A perfect snap for bioimage analysis," IEEE Signal Process. Mag., vol. 32, no. 1, pp. 41-48, Jan. 2015.

[6] B. Li and S. T. Acton, "Active contour external force using vector field convolution for image segmentation," IEEE Trans. Image Process., vol. 16, no. 8, pp. 2096-2106, Aug. 2007.

${ }^{2}$ http://icy.bioimageanalysis.org/plugin/Shape_Designer/ 
[7] T. McInerney and D. Terzopoulos, "Deformable models in medical image analysis: A survey," Med. Image Anal., vol. 1, no. 2, pp. 91-108, Jun. 1996.

[8] A. Jain, Y. Zhong, and M.-P. Dubuisson-Jolly, "Deformable template models: A review," Signal Process., vol. 71, no. 2, pp. 109-129, Dec. 1998.

[9] P. Thévenaz, R. Delgado-Gonzalo, and M. Unser, "The ovuscule," IEEE Trans. Pattern Anal. Mach. Intell., vol. 33, no. 2, pp. 382-393, Feb. 2011

[10] D. Schmitter et al., "A 2D/3D image analysis system to track fluorescently labeled structures in rod-shaped cells: Application to measure spindle pole asymmetry during mitosis," Cell Division, vol. 8, no. 6 , pp. 1-13, Apr. 2013.

[11] M. Jacob, T. Blu, and M. Unser, "Efficient energies and algorithms for parametric snakes," IEEE Trans. Image Process., vol. 13, no. 9, pp. 1231-1244, Sep. 2004.

[12] P. Brigger, J. Hoeg, and M. Unser, "B-spline snakes: A flexible tool for parametric contour detection," IEEE Trans. Image Process., vol. 9, no. 9, pp. 1484-1496, Sep. 2000.

[13] R. Delgado-Gonzalo, P. Thévenaz, C. S. Seelamantula, and M. Unser, "Snakes with an ellipse-reproducing property," IEEE Trans. Image Process., vol. 21, no. 3, pp. 1258-1271, Mar. 2012.

[14] L. Piegl and W. Tiller, The NURBS Book, 2nd ed. Berlin, Germany: Springer-Verlag, 2010.

[15] G. Charpiat, P. Maurel, J.-P. Pons, R. Keriven, and O. Faugeras, "Generalized gradients: Priors on minimization flows," Int. J. Comput. Vis., vol. 73, no. 3, pp. 325-344, Jul. 2007.

[16] X. Bresson, S. Esedoḡlu, P. Vandergheynst, J.-P. Thiran, and S. Osher, "Fast global minimization of the active contour/snake model," J. Math. Imag. Vis., vol. 28, no. 2, pp. 151-167, Jun. 2007.

[17] M. E. Leventon, W. E. L. Grimson, and O. Faugeras, "Statistical shape influence in geodesic active contours," in Proc. IEEE Conf. Comput. Vis. Pattern Recognit. (CVPR), Hilton Head Island, SC, USA, Jun. 2000 pp. $316-323$.

[18] M. Gastaud, M. Barlaud, and G. Aubert, "Combining shape prior and statistical features for active contour segmentation," IEEE Trans. Circuits Syst. Video Technol., vol. 14, no. 5, pp. 726-734, May 2004.

[19] Y. Chen et al., "Using prior shapes in geometric active contours in a variational framework," Int. J. Comput. Vis., vol. 50, no. 3, pp. 315-328, Dec. 2002.

[20] A. Foulonneau, P. Charbonnier, and F. Heitz, "Affine-invariant geometric shape priors for region-based active contours," IEEE Trans. Pattern Anal. Mach. Intell., vol. 28, no. 8, pp. 1352-1357, Aug. 2006.

[21] M. A. Charmi, S. Derrode, and F. Ghorbel, "Fourier-based geometric shape prior for snakes," Pattern Recognit. Lett., vol. 29, no. 7, pp. 897-904, 2008.

[22] F. de Chaumont et al., "Icy: An open bioimage informatics platform for extended reproducible research," Nature Methods, vol. 9, no. 7, pp. 690-696, Jul. 2012.

[23] M. Unser, "Sampling-50 years after Shannon," Proc. IEEE, vol. 88, no. 4 , pp. $569-587$, Apr. 2000

[24] M. Jacob, T. Blu, and M. Unser, "Sampling of periodic signals: A quantitative error analysis," IEEE Trans. Signal Process., vol. 50, no. 5, pp. 1153-1159, May 2002.

[25] R. Delgado-Gonzalo, P. Thévenaz, and M. Unser, "Exponential splines and minimal-support bases for curve representation," Comput. Aided Geometric Design, vol. 29, no. 2, pp. 109-128, Feb. 2012.

[26] M. Unser, "Splines: A perfect fit for signal and image processing," IEEE Signal Process. Mag., vol. 16, no. 6, pp. 22-38, Nov. 1999.

[27] T. Blu and M. Unser, "Quantitative Fourier analysis of approximation techniques. I. Interpolators and projectors," IEEE Trans. Signal Process., vol. 47, no. 10, pp. 2783-2795, Oct. 1999.

[28] I. J. Schoenberg, "Spline functions and the problem of graduation," Proc. Nat. Acad. Sci. United States Amer., vol. 52, no. 4, pp. 947-950, Oct. 1964.

[29] V. Uhlmann, R. Delgado-Gonzalo, C. Conti, L. Romani, and M. Unser, "Exponential Hermite splines for the analysis of biomedical images," in Proc. 39th IEEE Int. Conf. Acoust., Speech, Signal Process. (ICASSP), Firenze, Italy, May 2014, pp. 1631-1634.

[30] A. Aldroubi, M. Unser, and A. Aldroubi, "Sampling procedures in function spaces and asymptotic equivalence with Shannon's sampling theory," Numer. Funct. Anal. Optim., vol. 15, nos. 1-2, pp. 1-21, May 1994.

[31] T. F. Cootes, G. J. Edwards, and C. J. Taylor, "Active appearance models," IEEE Trans. Pattern Anal. Mach. Intell., vol. 23, no. 6, pp. 681-685, Jun. 2001.

[32] A. P. Britto and G. Ravindran, "Review of deformable curves-A retro analysis," Inf. Technol. J., vol. 6, no. 1, pp. 26-36, Jan. 2007.
[33] D. Jayadevappa, S. S. Kumar, and D. S. Murty, "Medical image segmentation algorithms using deformable models: A review," IETE Tech. Rev., vol. 28, no. 3, pp. 248-255, May 2011.

[34] W. H. Press, S. A. Teukolsky, W. T. Vetterling, and B. P. Flannery, Numerical Recipes: The Art of Scientific Computing, 3rd ed. Cambridge, U.K.: Cambridge Univ. Press, 1986.

[35] D. Eberly, R. Gardner, B. Morse, S. Pizer, and C. Scharlach, "Ridges for image analysis," J. Math. Imag. Vis., vol. 4, no. 4, pp. 353-373, Dec. 1994.

[36] A. F. Frangi, W. J. Niessen, K. L. Vincken, and M. A. Viergever, "Multiscale vessel enhancement filtering," in Medical Image Computing and Computer-Assisted Interventation-MICCAI (Lecture Notes in Computer Science), vol. 1496, W. Wells, A. Colchester, and S. Delp, Eds. Berlin, Germany: Springer-Verlag, 1998, pp. 130-137.

[37] M. Jacob and M. Unser, "Design of steerable filters for feature detection using canny-like criteria," IEEE Trans. Pattern Anal. Mach. Intell., vol. 26, no. 8, pp. 1007-1019, Aug. 2004.

[38] P. Ramdya and R. Benton, "Evolving olfactory systems on the fly," Trends Genet., vol. 26, no. 7, pp. 307-316, 2010.

[39] T. F. Cootes, C. J. Taylor, D. H. Cooper, and J. Graham, "Active shape models-their training and application," Comput. Vis. Image Understand., vol. 61, no. 1, pp. 38-59, Jan. 1995

[40] R. Delgado-Gonzalo and M. Unser, "Spline-based framework for interactive segmentation in biomedical imaging," IRBM, vol. 34 , no. 3 , pp. 235-243, Jun. 2013.

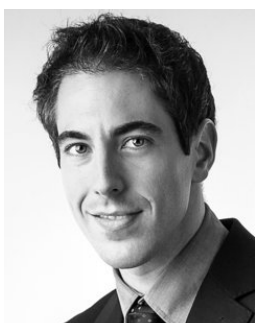

Ricard Delgado-Gonzalo received the Diploma degrees in telecommunications engineering and mathematics from the Universitat Politècnica de Catalunya (UPC), in 2006 and 2007, respectively, and the Ph.D. degree in biomedical image processing from the École Polytechnique Fédérale de Lausanne (EPFL), Switzerland, in 2013. In 2008, he joined the Biomedical Imaging Group, EPFL. He currently works on the design of highly efficient image-analysis open software capable of running on portable devices with an intuitive interface oriented to final users. He received the SSBE Research Award 2013 for the best Ph.D. thesis from the Swiss Society for Biomedical Engineering and the ABB Award 2014 from Asea Brown Boveri Ltd., for his contributions to the field of bioimage informatics.

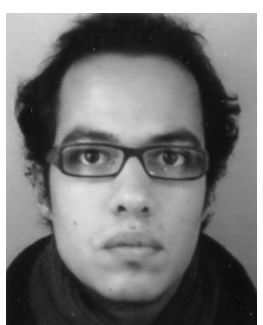

Daniel Schmitter received the master's degree in bioengineering and biomedical technologies from the École Polytechnique Fédérale de Lausanne (EPFL), Switzerland, in 2013, where he is currently pursuing the Ph.D. degree with the Biomedical Imaging Group. He was with the Advanced Clinical Imaging Technology Group, Siemens, at the Center for Biomedical Imaging, Switzerland, where he was one of the main contributors working on brain-imaging software and related image-processing algorithms. He is also involved in spline-based shape representation and segmentation problems. $\mathrm{He}$ has developed several segmentation and tracking software in biomedical imaging. 


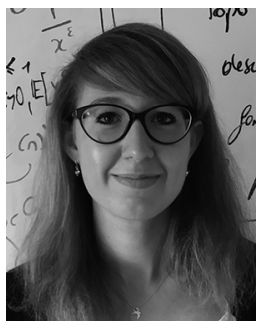

Virginie Uhlmann was born in Nyon, Switzerland. She received the M.S. degree in bioengineering from École Polytechnique Fédérale de Lausanne (EPFL), Switzerland, in 2012, where she is currently pursuing the $\mathrm{Ph} . \mathrm{D}$. degree with the Biomedical Imaging Group, under the direction of M. Unser. She pursued the master's thesis as a Visiting Student in the Imaging Platform with the Broad Institute, Cambridge, MA, under the supervision of A. Carpenter. From 2011 to 2012, she received the competitive Excellence Fellowship at the master's level from EPFL. She also received four prizes, including third best grade point average among EPFL's M.S. graduates of 2012. Her main research interests are applied problem related to bioimage segmentation, and in particular, spline-based methods, but also include computer vision, machine learning, and life sciences.

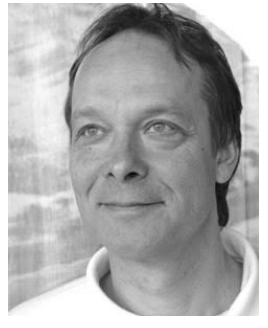

Michael Unser (M'89-SM'94-F'99) was with the Biomedical Engineering and Instrumentation Program, National Institutes of Health, Bethesda, USA, from 1985 to 1997, conducting research on bioimaging. He is currently a Professor and the Director of the EPFL's Biomedical Imaging Group, Lausanne, Switzerland. His primary area of investigation is biomedical image processing. He is internationally recognized for his research contributions to sampling theory, wavelets, the use of splines for image processing, stochastic processes, and computational bioimaging. He has authored over 250 journal papers on those topics. He has author the book An Introduction to Sparse Stochastic Processes with P. Tafti, (Cambridge University Press, 2014). He is a EURASIP Fellow (2009), and a member of the Swiss Academy of Engineering Sciences. He was a recipient of several international prizes, including three IEEE-SPS Best Paper Awards and two Technical Achievement Awards from the IEEE (2008 SPS and EMBS 2010). He is currently a member of the Editorial Board of SIAM Journal on Imaging Sciences, the IEEE Journal ON SELECTED TOPICS IN SignaL Processing, and Foundations and Trends in Signal Processing. He is the founding Chair of the Technical Committee on Bio Imaging and Signal Processing of the IEEE Signal Processing Society. He has held the position of the Associate Editor-in-Chief (2003-2005) for the IEEE TRANSACTIONS ON MEDICAL IMAGING. 